\title{
Goal Driven Service Composition for Online Travel Planning
}

\author{
V.Portchelvi \\ Research Scholar \\ Dept. of Banking \\ Technology \\ Pondicherry University \\ Pondicherry, South India
}

\author{
V. Prasanna \\ Venkatesan \\ Associate Professor \\ Dept. of Banking \\ Technology \\ Pondicherry University \\ Pondicherry, South India
}

\author{
G.Shanmuga \\ sundaram \\ Associate Professor \\ Department of IT
}

SMVEC

Pondicherry, South India
P.Sengamala Priya, J.Sharmila, R.Surya UG students

Department of IT

SMVEC

Pondicherry, South India

\begin{abstract}
To meet changing demands in the business process, multiple services have to be combined. Service Oriented Architecture offers Service Composition mechanisms, using which various services can be combined or composed according to user requirements. The services are combined in static and dynamic manner. The dynamic service composition approaches are moving towards goal driven technique which has gained more attention in recent days. Goal driven approaches reduce the users work by making the system to act dynamically according to the users requirements from the available set of services. The objective of the work mainly focuses on how to provide automated business process to meet the varying needs of the user. To explain the above mentioned approach travel domain is taken into consideration. Hence the outcome of our work in travel domain is to ensure that the goal driven service composition mechanism provides a complete travel trip plan.
\end{abstract}

\section{General Terms}

Software Architecture, SOA, Service-oriented Computing, AI Planning.

\section{Keywords}

Service composition technique, dynamic service composition, automated service composition, Goal driven service composition technique.

\section{INTRODUCTION}

Service Oriented Architecture is an architectural style that helps enterprises in integrating the business process so as to access the information over the network. SOA defines how two computing entities such as services interact and how one entity performs the work on behalf of another entity. These interactions between the services are performed using description languages.SOA helps businesses to move out from their monolithic structure and combine various applications in order to deliver a required complete service [7]. The main attraction of SOA is to provide a low cost infrastructure for the enterprises and to keep a step higher in the competitive environment by providing reusability. Service composition mainly came into existence to achieve a goal which mostly cannot be achieved through standalone service. A service composition is a process where n number of services can communicate with each other in order to satisfy the business needs [10]. Service composition provides a open standard based approach to connect the services in order to create higher level business process. Standards are designed in order to reduce the complexity required to compose the services, hence reducing the time and cost and increasing the overall efficiency. There are two approaches in service composition: static and dynamic service composition. In static service composition gathering of all the services are done at the design time and the composition of the components is first determined, obligated and finally expanded.

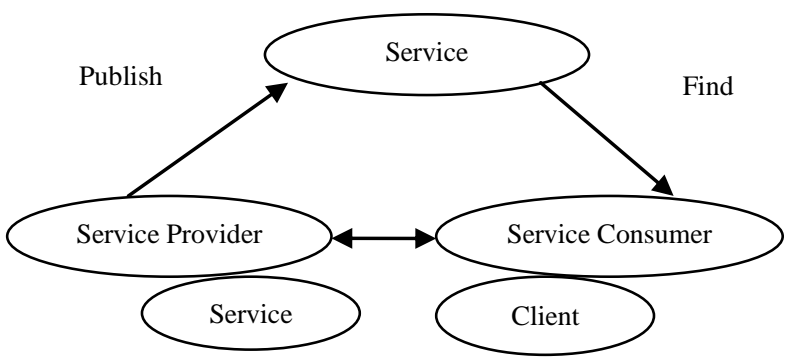

Fig 1: Service Oriented Architecture

This is possible only when the business is said to be static and if their features are unlikely to change. Static service composition is not adaptive to the changing requirements of the user.

Dynamic service composition was introduced in order to overcome the drawback of static service composition [5]. In dynamic service composition the composition can be made adaptable to any changing user requirements and it is also made flexible to the runtime changes. The environment in which service composition is done is highly dynamic and the numbers of service providers are increasing constantly leading to new services. The following section will provide the detailed description about the techniques used in composition.

\section{COMPOSITION TECHNIQUE}

There are many techniques being followed for composition of services [10]. But this section discusses in detail only about the AI planning based approach which comes under dynamic service composition.

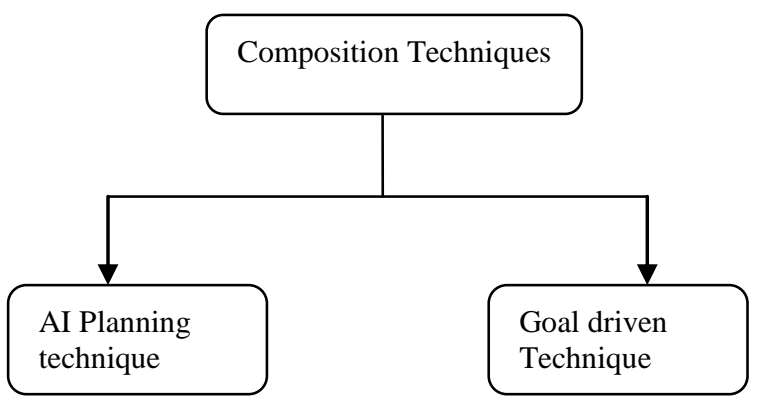

Fig 2: Composition Techniques 
As mentioned in previous section dynamic service composition drives the system in automated manner and makes it adapt to the changing clients requirements.

The AI planning considers a main technique named Goal Driven Service Composition which is not been implemented successfully yet.

\subsection{AI planning based composition:}

Automatic composition of service is not available completely at present and so to achieve this AI planning technique can be used [2]. The main element in automated service composition is goal. Goal is stated as set of actions performed in order to solve client's problem. Goal driven action planning is considered and an abstract goal is resolved automatically. The user specifies the objectives to be achieved in client side and AI planning algorithm takes the requirements of user and produces a plan based on that. In AI planning [10] the technical users feel convenient to use the goal as they are familiar with the initial states and final states .Intelligent algorithms are used in this technique which decides the initial and final states that cannot be easily understood by the non technical people/clients and hence for better understanding of the goal, based on users requirements goal driven service composition came into consideration.

\subsection{Goal driven service composition}

The goal carries all the necessary information required for discovering and composing the services. The goal driven service composition mainly focuses on meeting all the clients' requirements under a single shell. This type of composition is nothing but which makes the system to undergo automation of business services [1]. Here automation refers to providing all services to clients in a handy manner. This is possible only by using this technique which drives the system to get the requirements of the user in the initial stage and process based on it. The resulting service is said to be automated and a composed service which reduces the clients interaction with the system [15]. Ultimately this approach leads to failure handling where if the requested service is not available an alternative is provided. Hence the single goal given by the user processes the system automatically and this is referred to as Goal Driven Service Composition. Section 3 discusses about the application areas of goal driven service composition and further explains it in detail.

\section{APPLICATION}

Each and every sector nowadays makes use of goal driven composition approach either partially or completely. The areas which mainly focus in goal driven service composition are

- Weather forecasting

- $\quad$ E-Banking

- E-Learning

- Health care systems

- Financial sectors

- Travel sectors

Among the above mentioned areas travel domain has gained much importance. There is a need for service composition in virtual travel agencies. From the surveys which was undergone it is very clear that for service composition most of the cases considered to understand it was virtual travel agencies. For understanding the goal driven service composition more clearly we further explore in travel domain.

\section{VIRTUAL TRAVEL AGENCY}

A travel agency is a field where public services related to a trip is provided to the customers either for personal purposes or any commercial or business purposes. From the case study it is very clear that for understanding the concept of dynamic service composition travel agencies are considered. To make this concept more comprehensive virtual travel agencies provide a detailed and demonstrative explanation [4],[9]. Hence virtual travel agencies are considered as case study. Travel agencies generally aim to provide services such as transport, accommodation, guidelines etc... But today's travel agencies provide it manually which can also be said that it makes the user to choose each service individually and manually. This can be seen in this [12] portal where the users are made to move from transport service to hotel service or to holiday package or any other service manually. And in such portals it restricts the user to a single service. It does not provide any alternatives.

This can be explained clearly with an example. Consider a scenario where the user wants to travel from Chennai to Delhi. In Existing portals the user has to enter through the mode of transport and they have provided the sufficient parameters such as source, destination, data and time. Suppose the user select the train as mode of transport, the system responds through set of available services. During festival seasons or some unexpected situations the required train service may not be available. The system simply sends the message to user as the service is not available. This makes the user think that no other services are available and cancel their trip or allows the user to go and choose an alternative mode manually (Flight or Ship Service). Here two different cases can be explained.

1. In case one, when the required service is not available the system does not provide any alternatives for the current situation.

2. In such situation the user has to find an alternative by repeatedly doing the same task.

This can further be explained as the existing portals [12] do not handle any failure that occurs and in same way it does not provide any alternative automatically. Actually the ultimate goal of the user is to get a trip arranged in a more reliable and flexible manner. But these two key features are unavailable in existing system because the architecture which is developed for such system does not contain that facility. Usually any trip planning agencies should provide a system with four layer namely user request layer, goal layer, task layer and service layer. But in today's portals the goal layer and task layer are binded together and such binding does allow the system to provide any alternative and handle the failure. So to overcome such drawbacks the proposed system was developed with a failure handing and an alternative providing facility.

\section{PROPOSED TECHNIQUE}

Our proposed system architecture is as follows in fig 3. The existing system architecture for travel domain consists of three process planning, execution and orchestration. These processes were not implemented completely in the existing system [3] as they failed to execute the layers present within them individually. Actually each process consists of various layers such as task layer, user request layer, goal layer, service layer. In the existing system the task layer and the goal layer are binded together which is the major reason for not providing alternatives. But in the proposed system the goal layer and the task layer are placed in separate processes planning and execution.

\subsection{Planning}

Planning is the process of providing a plan for a complete trip to the user based on his/her requirements. This is very essential phase because it includes obtaining all the needs of the user at this phase based on which the entire system is going to progress. It comprises of the user request layer and the goal layer. These layers play a major role in planning a trip by maintaining the requirements of the client throughout the process. 


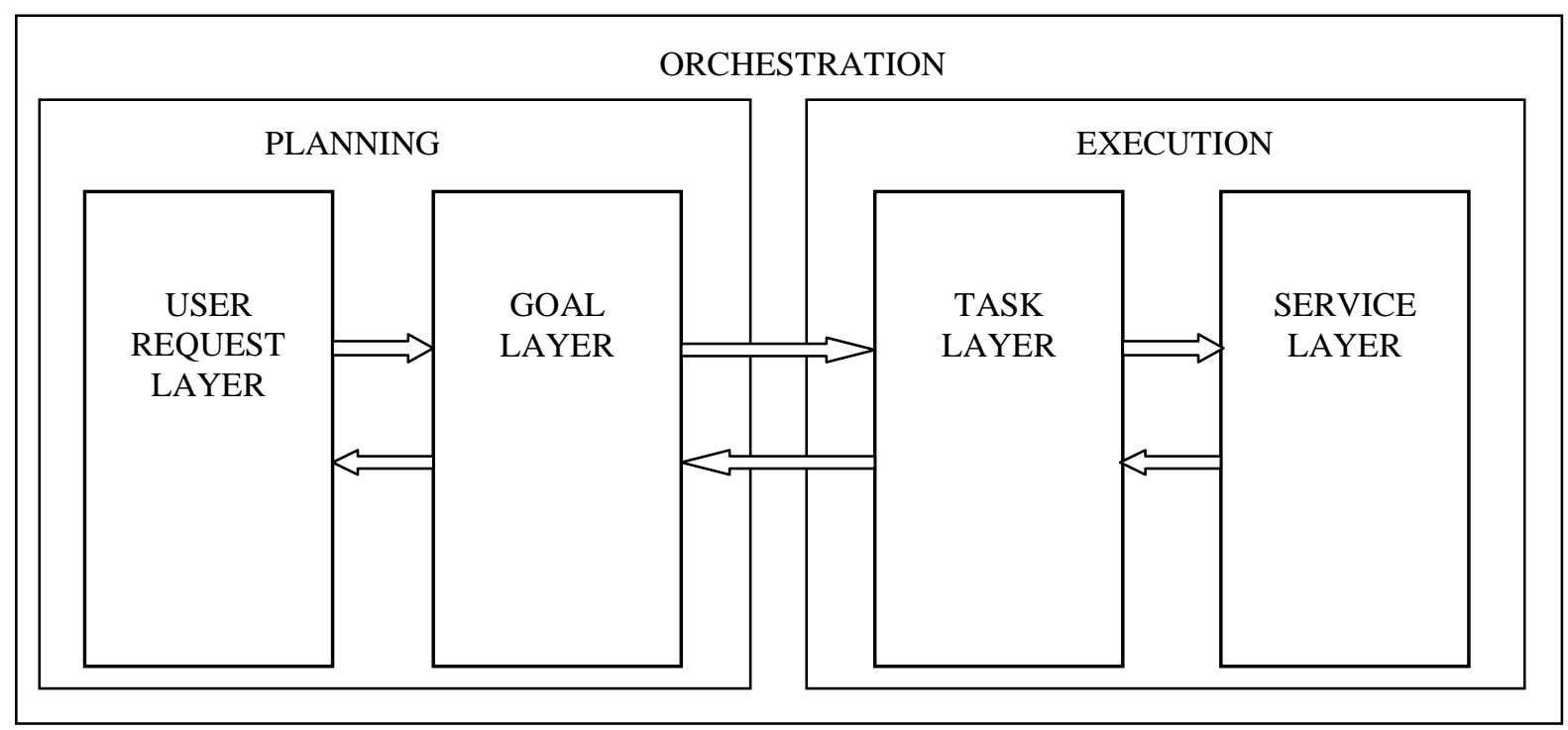

Fig 3: Architecture for proposed travel system

\subsubsection{User request layer}

The initial layer refers to the user request layer. In our plan this is the layer the user sees first. In this layer user needs to enter the required preferences for the entire trip. So this layer is very important as it contains all the necessary information for the full process.

\subsubsection{Goal layer}

This layer is the place where the system plan moves based on the information entered by the user. The decision is made in this layer. Goal layer here is separated from the task layer so that if any failure occurs the backtracking will not proceed till user request layer. This saves from getting the details of the user again.

\subsection{Execution}

This part mainly executes the plan that was made in the planning phase. The execution phase collects the details from the planning phase and executes it in such a way that the entire trip is processed. It consists of two layer task layer and service layer.

\subsubsection{Task layer}

This layer describes the services in an abstract manner. In our plan for example if the user enters the source and destination, the keywords are matched with the database and services such as bus or train or flight is provided to the user . This is abstract layer.

\subsubsection{Service layer}

This layer is more concrete it provides all the features in a particular service .For example if bus is the mode of transport chosen then the concrete details about the bus such as bus name, arrival and departure time, seats availability, etc, are provided.

\subsection{Orchestration}

Here orchestration is the main feature which combines the planning and execution phases. An orchestrator is the person who co-ordinates the work and manages the process. Similarly orchestration is the phase which combines both the phases and manages the overall execution. Orchestration mainly focuses on processing the services automatically. This feature drives our goal driven service composition technique in the proposed system.

\subsection{Working of proposed system}

In proposed system initially it allows the user to login. If he is a new user then a registration is required. After the registration user is asked for preferences for the entire trip planning. This is used in future also when the user login next time. Facilities are also provided to change it if required. These preferences are got for the entire trip. Then the source and destination along with date and times is given. The user can also provide any particular mode of transport if required. Then based on the users request and preferences first the mode of transport is searched. The services which are available are fetched and placed separately in a database named services. Similarly this is also performed for the hotel (i.e.) accommodation. Based on the preferences and users request available services of hotels are searched and put in same services table. Finally all the available services requested by the user are displayed. If in middle of any service processing failure occurs the system provides an alternative suggestion to the user. Finally the services displayed are provided as separate services whose link can be by the users to complete their trip planning. The graph explains our trip plan. The trip planning consists of transport, accommodation and a local transport. This layer is referred to as the user request layer. The next layer where the user preferences are saved is the goal layer and the task layer here is the bus, train, flight and in accommodation it is hotels, lodges etc. Each service has various sub division which can be compared with the user preferences. So the tree proceeds till the final user request is obtained. The next section shows the comparison between the existing and proposed systems.

\subsection{Advantage of proposed system}

The architecture in section 5 clearly shows that the goal layer and task layer are separated. This makes the system to provide alternatives without human intervention. That is in existing system when a user request is given and the system performs according to the instructions but if any failure occurs the system backtracks till the user request and gets the user request again. But in our architecture since the goal and task layer are separated it has reduced the user work. This is because the user request is obtained again from the goal layer and the user need not enter the request again. This advantage reduces most of the user work and makes it an automatic system. The following section is the comparison made between the existing and the proposed system. 


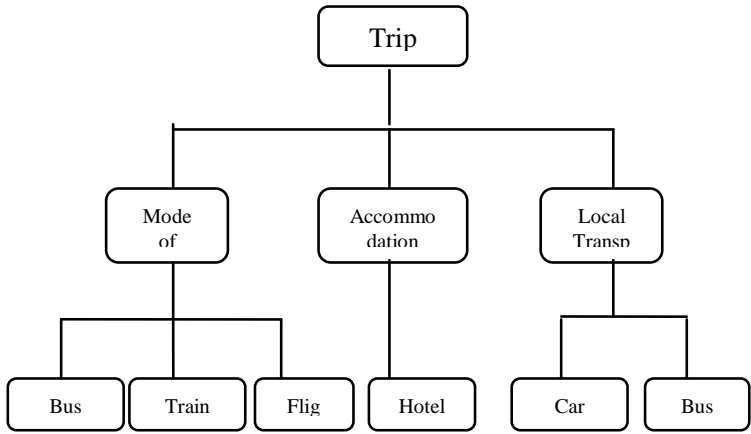

Fig 4: Tree for developed travel system

\section{DISCUSSION}

This discussion helps us to understand clearly how our system varies from existing system [14]. The comparison is based on various parameters such as accessibility, providing alternatives, failure handling [8]. To explain briefly let us consider the first factor accessibility. In consider to book a ticket for Chennai to Delhi. The user chooses mode of transport as bus. But in real time buses are not available to Delhi. The system shows an exception after which no alternative is been provided to the user by the system for which he/she has to find an alternative solution. But in our proposed system if a failure occurs automatically it moves to an alternative. So the human intervention is reduced here. This saves a lot of time. Failure handling is present in every system. But the way of handling it intelligently is not available in today's travel systems. But our system which is developed handles any failure easily by providing much number of solutions to the required requirements. An alternative is the main need which the user requires when any failure occurs in the system. Such alternatives are not provided automatically in existing system. This was a major drawback which was overcome in our proposed system where system provides various alternatives. The following table gives a clear understanding:

Table 1 Comparison of Proposed And Existing Approaches

\begin{tabular}{|l|l|l|}
\hline PARAMETERS & $\begin{array}{l}\text { EXISTING } \\
\text { SYSTEM }\end{array}$ & $\begin{array}{l}\text { PROPOSED } \\
\text { SYSTEM }\end{array}$ \\
\hline Failure handling & Not possible & Possible \\
\hline Alternatives & Manual & Automatic \\
\hline
\end{tabular}

\section{CONCLUSION}

The possibilities of approaches for automatic service composition are analyzed by giving more attention to goaldriven service composition. The analysis shows that there is a lack of approaches that are used for achieving goal driven dynamic service composition in travel domain. Most of the approaches are focused on providing a static service composition. Despite many papers provided approaches for dynamic service composition it does not give a clear clarification about the goal driven service composition.

We showed in our system that dynamic service composition can be achieved automatically using goal driven approach. An example is also provided in travel domain to show how the system achieves dynamic service composition and how it handles exception handling.

\section{REFERENCES}

[1] Michael Stollberg, Martin Hepp , July 10, 2006, WP3: Service Ontologies and Service Description D3.10 Goal Description Ontology.

[2] Sudhir Agarwal, 2007, A Goal Specification Language for Automated Discovery and Composition of Web Services, in Proc. of International Conference on Web Intelligence, 2007 IEEE/WIC/ACM.

[3] Wu, D., Sirin, E., Hendler, J., Nau, D., and Parsia, B. 2003. "Automatic Web Services Composition Using SHOP2". Twelfth World Wide Web Conference.

[4] P. Traverso \& M. Pistore ,2004, "Automated Composition of Semantic Web Services into Executable Processes". In: Proc. of ISWC-04, LNCS 3298. Springer, pp. 380-394.

[5] Peer, J., 2005 "Web service composition as AI planning a survey". Technical report, Univ. of St. Gallen, Switzerland.

[6] Juan Carlos Zũniga, Jos'e J. P'erez-Alc'azar, Luciano Digiampietri, 2010, "Implementation Issues for Automatic Composition of Web Services", 2010 Workshops on Database and Expert Systems Applications.

[7] M. P. Papazoglou et al., 2007,'’Service-Oriented Computing: State of the Art and Research Challenges, Computer", vol. 40, no. 11, pp. 38-45.

[8] Biplav Srivastava and Jana Koehler, 2003, "Web service composition - current solutions and open problems, in Proc. Of International conference on Automated Planning and Scheduling".

[9] Dalè Dzemydienè and Arūnas Miliauskas, ISSN 1392 0561. INFORMACIJOS MOKSLAI. 201365 "Possibilities of automatic and semi-automatic end-user driven web service composition".

[10] Peer, J.,2005, "Web service composition as AI planning - a survey”, Univ. of St. Gallen, Switzerland, Technical report.

[11] Rao. J. and X. Su,2004, "A Survey of Automated Web Service Composition Methods", in Proceedings of the First International Workshop on Semantic Web Services Web Process Composition,.

[12] www.makemytrip.com

[13] Marco Pistore , Jose Luis Ambite, Jim Blythe, Jana Koehler, Sheila McIlraith, Biplav Srivastava,2006, "AI for Service Composition", in workshop .

[14] Hua Xiao, Ying Zou, Ran Tang, Joanna Ng, Leho Nigul,2009, "An Automatic Approach for Ontology-Driven Service Composition".

[15] S. Dustdar, W. Schreiner,2005, "A survey on web services composition", Int. Journal of Web and Grid Services, vol. 1 , no.1, pp. 1-30. 